\title{
Chinese Privacy Law: A Practitioner's Guide to Current Regulations, Future Trends, \& Business Applications
}

\author{
Tiffany Li \& Zhou Zhou ${ }^{1}$ \\ Updated January 13, 2016
}

\footnotetext{
${ }^{1}$ Tiffany Li is Privacy Fellow, Wikimedia Foundation; J.D., Georgetown University Law Center; B.A., University of California, Los Angeles. Zhou Zhou is Legal Counsel, Wikimedia Foundation; J.D., Columbia Law School; B.S., University of California, Berkeley. The views expressed in this Article do not necessarily reflect the views of our employer or any other organization. A version of this Article was published in serial form in "The Privacy Advisor" (International Association of Privacy Professionals, 2015).
} 
The business case for understanding Chinese privacy law is clear. With China's growing economic power and large consumer base, any international company seeking to profit from consumers in the region should expend resources on understanding how best to succeed in the Chinese market. This means that privacy officers must pay close attention to how privacy law is developing in China, both to keep up with current developments and to stay ahead of the curve by proactively implementing strategic policies.

This Article will provide a basic privacy practitioner's guide to privacy law in China. First, we will go over the basic status of privacy laws and regulations in China. Second, we will delve into the cultural and historical factors that influence the development and application of Chinese privacy law. Finally, we will offer practical lessons and hypothetical case studies for how to proactively help your company or organization succeed in China.

\section{Current State of Privacy Laws in China}

Privacy law in China is still a relatively new field. While China lacks a comprehensive national privacy law, there are a number of privacy laws and regulatory guides focused on specific concerns.

To understand the current state of Chinese privacy laws, it's best to first distinguish the concepts of privacy from government actors and privacy from corporate actors. China's current legal system does not afford significant privacy protections against government intrusion. However, in the past few years, China has been increasingly willing to create and enforce privacy laws aimed at regulating corporations and private actors. 
In the past few years, China has created a number of privacy laws focused on regulating the private sector. These laws include telecommunications regulations like the 2011 MIIT "Internet Information Services Regulations" (“MIIT 2011 Regulation")'; the 2013 MIIT

"Telecommunications and Internet Personal User Data Protection Regulations" ("MIIT 2013 Regulation") ${ }^{3}$; the 2013 MIIT "Information Security Technology - Guidelines for Personal Information Within Public and Commercial Services Systems," ("MIIT 2013 Guide"). Additionally, in 2012, the SC-NPC Decision on Internet Information Protection ("SC-NPC $2012 ")^{5}$ created the highest level law in China dealing with data protection. The provisions from SC-NPC 2012 were largely included in the 2013 amendments to China's "Law on the Protection of Consumer Rights and Interests" ("Consumer Law"). ${ }^{6}$

The amended Consumer Law includes the general principle that all operators must "follow legal, legitimate, and necessary principles," incorporating principles stated in the SC-NPC 2012. The Consumer Law (2013), Art. 29 lists these basic principles:

"Proprietors collecting and using consumers' personal information shall abide by principles of legality, propriety and necessity, explicitly stating the purposes, means and scope for collecting or using information, and obtaining the consumers' consent.

\footnotetext{
${ }^{2} 2011$ MIIT “Internet Information Services Regulations" ("MIIT 2011 Regulation”), http://www.miit.gov.cn/n11293472/n11293832/n12771663/14417081.html.

${ }^{3} 2013$ MIIT "Telecommunications and Internet Personal User Data Protection Regulations" ("MIIT 2013 Regulation"), https://chinacopyrightandmedia.wordpress.com/2013/07/16/telecommunications-and-internet-userindividual-information-protection-regulations/.

${ }^{4} 2013$ MIIT "Information Security Technology - Guidelines for Personal Information Within Public and Commercial Services Systems," ("MIIT 2013 Guide"). ${ }^{4}$ http://www.pipa.gov.cn/manage/UploadFile/2015518133720563.pdf.

${ }^{5}$ SC-NPC Decision on Internet Information Protection ("SC-NPC 2012"), https://chinacopyrightandmedia.wordpress.com/2012/12/28/national-peoples-congress-standing-committeedecision-concerning-strengthening-network-information-protection/.

${ }^{6} 2013$ amendments to China's "Law on the Protection of Consumer Rights and Interests" ("Consumer Law"), http://chinalawtranslate.com/consumer-protection-law-including-2013-amendments/?lang=en.
} 
Proprietors collecting or using consumers' personal information shall disclose their rules for their collection or use of this information, and must not collect or use information in violation of laws, regulations or agreements between the parties.

Proprietors and their employees must keep consumers' personal information they collect strictly confidential and must not disclose, sell, or illegally provide it to others. Proprietors shall employ technical measures and other necessary measures to ensure information security, and to prevent consumers' personal information from being disclosed or lost. In situations where information has been or might be disclosed or lost, proprietors shall immediately adopt remedial measures.

Proprietors must not send commercial information to consumers without their consent or upon their request of consumers, or where they have clearly refused it."

These principles are echoed in the general principles of MIIT 2011, which state that ISPs "shall provide services in accordance with the principles of equality, free will, fairness and good faith.”

In 2013, the MIIT released a voluntary guide for industry best practices. The MIIT 2013 Guide elucidates eight principles, which are all fairly standard for international privacy norms and similar to other nations' privacy principles:

1. Clear Purpose Principle: Data should only be used for a clear purpose and should not be used outside of that purpose. 
2. Minimum and Sufficiency Principle: Companies should only handle the minimum amount of information sufficient for their purpose. Personal info should be deleted after the purpose has been achieved.

3. Public Notification Principle: Companies should give notice to data subjects of the purpose, scope, use, security, and other information related to the data collection.

4. Personal Consent Principle: Companies should obtain consent before using data.

5. Quality Assurance Principle: Companies should take measures to ensure confidentiality, integrity, availability of data.

6. Safety Guarantee Principle: Companies should take measures to ensure security of data.

7. Good Faith Fulfilling Principle: Companies should act in good faith in compliance with legal requirements.

8. Clear Responsibility Principle: Companies should clearly define responsibilities, taking of appropriate measures, and records processing (for use in potential future investigations).

These principles should be familiar to any privacy practitioner. Additionally, the MIIT 2013 Guide includes the following: special safeguards for sensitive information, restrictions for overseas data exports, data breach notification obligations, and information on rights of data subjects. The MIIT 2013 Guide is the only current state policy that mentions limits on transnational data exportation, explicitly prohibiting overseas transfer of data without express consent of data subjects or explicit legal or regulatory permission. However, the MIIT 2013 Guide is voluntary and does not impose legal obligations upon corporations. 
Chinese citizens may have a right to privacy under tort law. China's General Principles of Civil Law ("GPCL") includes protection of the "right of reputation" (GPCL, art. 101), while the Tort Liability Law ("TTL") protects the "right of privacy" (TTL, art. 2). Both the GPCL and TTL tort rights of privacy have been used in litigation between individuals and corporations. It is important to note that, under the TTL, "network service providers" (likely to be interpreted as similar to "Internet Service Providers" in the West) have special obligations to prevent tortious actions committed by users of their services.

China also has some privacy-focused sectorial legislation (laws governing specific industries), somewhat similar to the U.S. sectorial approach to privacy law. China's sectorial laws include the Basic Norms for Electronic Medical Records (Ministry of Health, 2010), Social Insurance Law (2010), and Regulations and Administrative Measures for Credit Reference Agencies (2013). In addition, a number of provinces and municipalities have enacted their own privacy laws.

In conclusion, while Chinese privacy law is still fairly undeveloped, China has made significant strides in recent years to create new laws governing data privacy and confidentiality of personal information. Some of the major principles inherent in the highest level Chinese privacy laws are similar to international privacy norms (E.U. Directive, APEC Principles, etc.). With the law still unclear, the best way forward for privacy practitioners is to ensure compliance with current laws and proactively adapt practices that are in touch with Chinese consumer culture.

\section{Cultural Factors Influencing the Perception of Privacy}


As is the case legal systems in all countries, Chinese culture affects Chinese views and application of laws - including privacy laws. However, we cannot study Chinese privacy law in a vacuum. The modern legal conception of privacy, and its applicable laws, arose from Western culture. Thus, to understand Chinese privacy law, we must juxtapose Western notions of privacy against thousands of years of Confucian teaching and the historical background that still influences present-day Chinese society. In fact, such cultural considerations have such a strong influence on Chinese privacy laws precisely because the importation of Western privacy laws prevented China from developing its own sui generis privacy culture.

Historically, Chinese laws arose out of Confucian ideas about government and have always been underpinned by Confucian ethics and morality. As such, Chinese laws and their applicability are dependent on Chinese cultural assumptions about ethical and moral behavior. International norms for privacy law are also based on ethical and moral considerations. Thus, any tensions between Western theories of privacy and the reality in the Chinese legal system can be particularly problematic for the implementation of privacy laws since both forces are based on strong, and sometimes conflicting, value systems.

In particular, it is possible that the deep importance of community in Chinese culture could impede the proper application of privacy laws in China. Confucian ideas arose thousands of years ago out of a time in Chinese history when the country was undergoing an extended and tumultuous civil war. During that turbulent time, prominent scholars, like Confucius, sought ways to end the turmoil. Thus, under Confucianism, and the Chinese legal systems based on it, priority is placed on ensuring order and good governance. In such a system, the rights of the individual are subordinate to the rights of the family unit, the community, and the country. Even 
today, many Chinese citizens almost implicitly acquiesce to the idea that any privacy rights they have are not absolute against the monitoring powers of the centralized state. Furthermore, in many recent privacy-related disputes in China, the legal system have often sided with protecting the rights, values, and morals of the community over protecting the privacy rights of the individual. $^{7}$

A second complication for privacy practitioners seeking to understand privacy in China is the strong contrast between Chinese and Western ideals involving regulation of individual behavior. In the Judeo-Christian Western culture, it's arguable that punishment is based on the personal and inward-facing ideas of individual wrongdoing and individual guilt. However, in Chinese culture, punishment often derives from the community-infused concept of Shame. As one commentator notes ${ }^{8}$, in this Shame-based culture, purported wrongdoers can only be properly punished if their faults are exposed to the community and the punished are forced by society to correct their behavior based on what society thinks is morally important to do. Under exposure to the criticism of their community, an individual's privacy rights are disregarded. This cultural artifact has contributed to the privacy-invasive phenomena of 'human-flesh' search engines ${ }^{9}$ that commonly engulf online communities in mainland China. ${ }^{10}$

\footnotetext{
${ }^{7}$ For a further discussion of such cases, please see 隐私权的中国命运一司法判例和法律文化的分析 by 张礼洪, 隐私权的中国命运一司法判例和法律文化的分析. http://www.cnki.com.cn/Article/CJFDTotal-SDFX201401002.htm. 
On the other hand, the shame-based culture means that in certain ways, Chinese citizens are very protective of their public image and any disclosure of private wrong-doing, whether legal or moral, that could lead to a loss of 'face.' This is likely one impetus behind the privacy and confidentiality laws that do exist in China today, and could point to the direction that Chinese privacy laws might further develop in the future.

The aforementioned mix of cultural factors has produced a unique Chinese view towards privacy - a view that is strict in certain respects but lax in others. Understanding these cultural and historical factors can offer clues to how Chinese privacy law may develop in the future. It is also helpful to keep these considerations in mind, when creating privacy practices that deal with Chinese consumers or Chinese corporations.

\section{Practical Lessons}

It is difficult to predict with any certainty how privacy law in China will develop. As we have already discussed, Chinese privacy laws are still in their early stages. There is no comprehensive national privacy law, and what laws do exist are generally similar to international norms (notice, choice, security, and so on).

Here are a few practical lessons to help you proactively protect your company and your clients from legal privacy risks in China.

\section{A. Designing the Best China-Centric Privacy Policies}


1. Focus on community values

Given the Chinese emphasis on community values, it is advisable to design your privacy policies and practices in context of an understanding of Chinese community values. For example, in framing a discussion of your policies, include a discussion of the values of your particular online community and explain how your privacy practices protect those values.

2. Look to ethics, not just law

Look to ethics first, not just the law. China does not yet have strong privacy laws, so the best basis for any privacy-related policy is probably ethics. Chinese consumers may also be particularly swayed by ethical arguments for privacy practices, given historical focus on ethical bases for laws. To put this in practice, include an ethical basis when justifying your privacy policies, in addition to providing the legal basis for your policies.

3. Use culture for context

Use culture for context. Different cultural values of privacy affect what your users want from corporations. In the U.S., users may view privacy almost as a property right (protecting private information a user owns). In China, users may instead view privacy as a shield, protecting private lives from community exposure or shame from losing face. Recognize this distinction in your policies by emphasizing how your company will protect the confidences of your users and prevent information exposure.

\section{B. Understanding Chinese Consumer Expectations}


1. Chinese consumers may have fewer privacy expectations

Chinese consumers may place less value on privacy, when compared to other competing values or obligations. This may be reflected in what your user community and consumers value. For example, your users may care more or be affected more by corporate transparency efforts than privacy protection efforts. Thus, you may receive a better return on investment for putting resources into creating and publishing transparency reports.

2. Chinese consumers do still care about privacy

At the same time, privacy practitioners should not assume that Chinese consumers do not care at all about privacy. Cultural expectations of privacy may be different, but privacy is still valued in China, especially privacy concerning confidentiality of personal information. Keep in mind the notions of "shame" and "saving face." It is important to remember that, while Chinese consumers may have a different conception of privacy rights, this is no excuse for not protecting the privacy of your consumers or failing to follow the law.

3. Chinese consumers may be more open to data collection/use Chinese consumers may be more pragmatic about companies using or buying their private information. If you can make a strong case for why your users should give their data (e.g., in exchange for tailored features), it is likely that Chinese consumers will be more swayed by these arguments than European consumers, for many of whom privacy is an almost inalienable right. Privacy is not a fundamental Chinese value, so Chinese 
consumers may be less tied to strict privacy rights, when compared to Western consumers.

Understanding the cultural and historical factors behind China's privacy laws today provides a path forward in creating policies and programs that will best engage Chinese consumers and likely minimize future legal compliance risks. These practical privacy lessons can be used when conducting due diligence on new Chinese business interests, creating new policies for overseas corporate expansion, or interacting with Chinese user communities.

As noted, Chinese privacy law is still fairly underdeveloped, and it is difficult to predict how the laws will change in the future. However, understanding the current state of Chinese privacy law, as well as the cultural and historical factors that might influence its development and application, will help privacy practitioners prepare their organizations to succeed in the growing Chinese market.

For further discussion on Chinese privacy law, and Asian privacy law in general, we recommend the comprehensive treatise, Asian Data Privacy Laws: Trade and Human Rights Perspectives, by University of New South Wales Law Professor Graham Greenleaf. 